\title{
Concurrent inhibition of IDH and methyltransferase maximizes therapeutic efficacy in IDH mutant acute myeloid leukemia
}

\author{
Zhihong Zeng ${ }^{1}$ and Marina Konopleva ${ }^{1,2}$ \\ ${ }^{1}$ Department of Leukemia, The University of Texas MD Anderson Cancer Center and ${ }^{2}$ Department of Stem Cell Transplantation and Cellular \\ Therapy, The University of Texas MD Anderson Cancer Center, Houston, TX, USA \\ E-mail: MARINA KONOPLEVA - mkonople@mdanderson.org
}

doi:10.3324/haematol.2020.266809

W

ith findings of the molecular mechanisms of cotargeting isocitrate dehydrogenase (IDH) and methyltransferase with IDH inhibitors and a hypomethylating agent (HMA), Chaturvedi et al. ${ }^{1}$ provide new insights into combination therapy for IDH mutant acute myeloid leukemia (AML). Their manuscript entitled "Synergistic activity of IDH1 inhibitor BAY1436032 with azacitidine in IDH1 mutant acute myeloid leukemia," published in this issue of Haematologica, reports that the concurrent administration of an IDH inhibitor and an HMA maximizes antileukemia efficacy in vitro and in vivo.

IDH is an enzyme that catalyzes conversion of isocitrate to $\alpha$-ketoglutarate ( $\alpha$-KG); its metabolism plays an essential role in balancing cellular energy. IDH1 and IDH2 are 2 of the 3 isoforms identified in humans; mutations in both isoforms are found in several malignancies, including AML. Mutant IDH1/2 converts $\alpha$-KG to 2-hydroxyglutarate (2-HG), a metabolite structurally similar to $\alpha-K G$, competitively inhibits $\alpha$-KG-dependent enzymes, ultimately alters DNA and histone methylation, and impairs cellular growth and differentiation. ${ }^{2,3}$ Recurrent IDH1/2 mutations occur in up to $20 \%$ of patients with AML. 4.5 In IDH mutant AML, overproduction of 2-HG leads to DNA and histone hypermethylation and myeloid cell differentiation arrest. ${ }^{6}$

Several small-molecule inhibitors of mutant IDH1 and IDH2 have been developed and are progressing through preclinical and clinical development.,8 Two of them, ivosidenib and enasidenib, are approved by the Food and Drug Administration (FDA) for the treatment of newly diagnosed and relapsed/refractory IDH1 or IDH2 mutant AML, respectively. BAY1436032 is an oral, pan-mutant IDH1 inhibitor characterized by researchers in this study. BAY1436032 has shown strong antileukemic activity in two separate IDH1 mutant AML xenograft mouse models. This compound is currently undergoing safety/efficacy testing in phase I dose escalation trials in patients with relapsed IDH1 mutant AML (clinicaltrials gov. Indentifier: 03127735) and advanced solid tumors (clinicaltrials gov. Indentifier: 02746081). ${ }^{10}$ Despite high efficacy and on-target activity, IDH-targeted monotherapy in AML offers response rates of less than 50\%. Resistance mechanisms, such as co-occurring mutations in receptor tyrosine kinase signaling (FLT3, PTPN11, RAS, KIT), transcription factors (RUNX1, GATA2, CEBPA) and restoration of 2-HG through mutations in other IDH protein or IDH1 second site mutations, may account for the therapeutic failure; therefore, combination regimens are essential to improve clinical responses. ${ }^{11,12}$ The combination of an IDH1/2 inhibitor (ivosidenib or enasidenib) with the HMA azacitidine is being evaluated in an ongoing phase Ib/II study in patients with newly diagnosed IDH mutant AML who are ineligible for intensive chemotherapy (clinicaltrials gov. Indentifier: 02677922). This combination was well tolerated, with a safety profile consistent with that of ivosidenib or azacitidine monotherapy.
Clinical response rates exceeded those of azacitidine alone, and importantly, most responders achieved IDH1 mutation clearance..$^{13}$ Based on these findings, a phase III study of ivosidenib and azacitidine is actively enrolling patients (clinicaltrials gov. Indentifier: 03173248). Despite these encouraging clinical responses, the molecular mechanisms of the interaction between IDH inhibitors and HMA are not well understood.

In this issue of Haematologica, Chaturvedi et al. ${ }^{1}$ investigated the efficacy and elucidated the mechanism of action of the novel IDH1 inhibitor BAY1436032 combined with azacitidine in both in vitro and in vivo preclinical models of IDH1 mutant AML. The authors observed that ex vivo treatment with BAY1436032 and azacitidine is more effective than singleagent treatment in its ability to induce the cell cycle $S$ phase block, resulting in synergistic inhibition of colony formation in primary IDH1 mutant AML. Using two human IDH1 mutant AML xenograft models, the team evaluated the efficacy of BAY1436032 and azacitidine as single agents and in combination, with sequential (azacitidine followed by BAY1436032) or concurrent applications in vivo. Combination therapy induced differentiation and significantly prolonged survival compared to single agent or control cohorts. Importantly, concurrent administration of these agents, similar to the design implemented in the ongoing clinical trials, produced the highest efficacy; this finding was further confirmed in a secondary transplantation assay indicating that the concurrent combination therapy elicits the greatest reduction in the number of leukemia stem cells (LSC).

Using transcriptomic (RNA sequencing) and epigenomic (DNA methylation arrays) analyses, the authors explored the mechanisms underlying this additive/synergistic efficacy, studying AML cells collected in vivo. Combination therapy decreased the expression of LSC gene sets and suppressed transcriptional factors in MAP kinase (RAS/RAF) and retinoblastoma/E2F (RB/E2F) pathways. Quantitative RTPCR analysis confirmed that the cell survival/proliferation genes ELK1, ETS1, and CCND1 in the MAP kinase pathway and E2F1, CCNA2, and CCNE1 in RB/E2F signaling were additively suppressed, while the myeloid differentiation genes PU.1, CEBPA, and GABPA were upregulated in cells retrieved from the combination therapy arm. Using an IDH1mutant fibrosarcoma cell line, the authors evaluated these findings at the translational level: the concurrently administered combination dephosphorylated ERK1/2 and downregulated the downstream targets of ELK1, ETS1 and CYCLIN D1. The lack of CYCLIN D1 limited CYCLIN D-CDK4 complex formation and consequently inhibited RB phosphorylation on serine 795 and 807/811, thereby preventing RB from releasing E2F to regulate cell cycle G1 to $\mathrm{S}$ transition (Figure 1). Correspondingly, directly targeting the MAP kinase pathway with a MEK1/2 inhibitor, trametinib, or blocking the cell cycle with a CDK4/6 inhibitor, abemaciclib, more effectively 


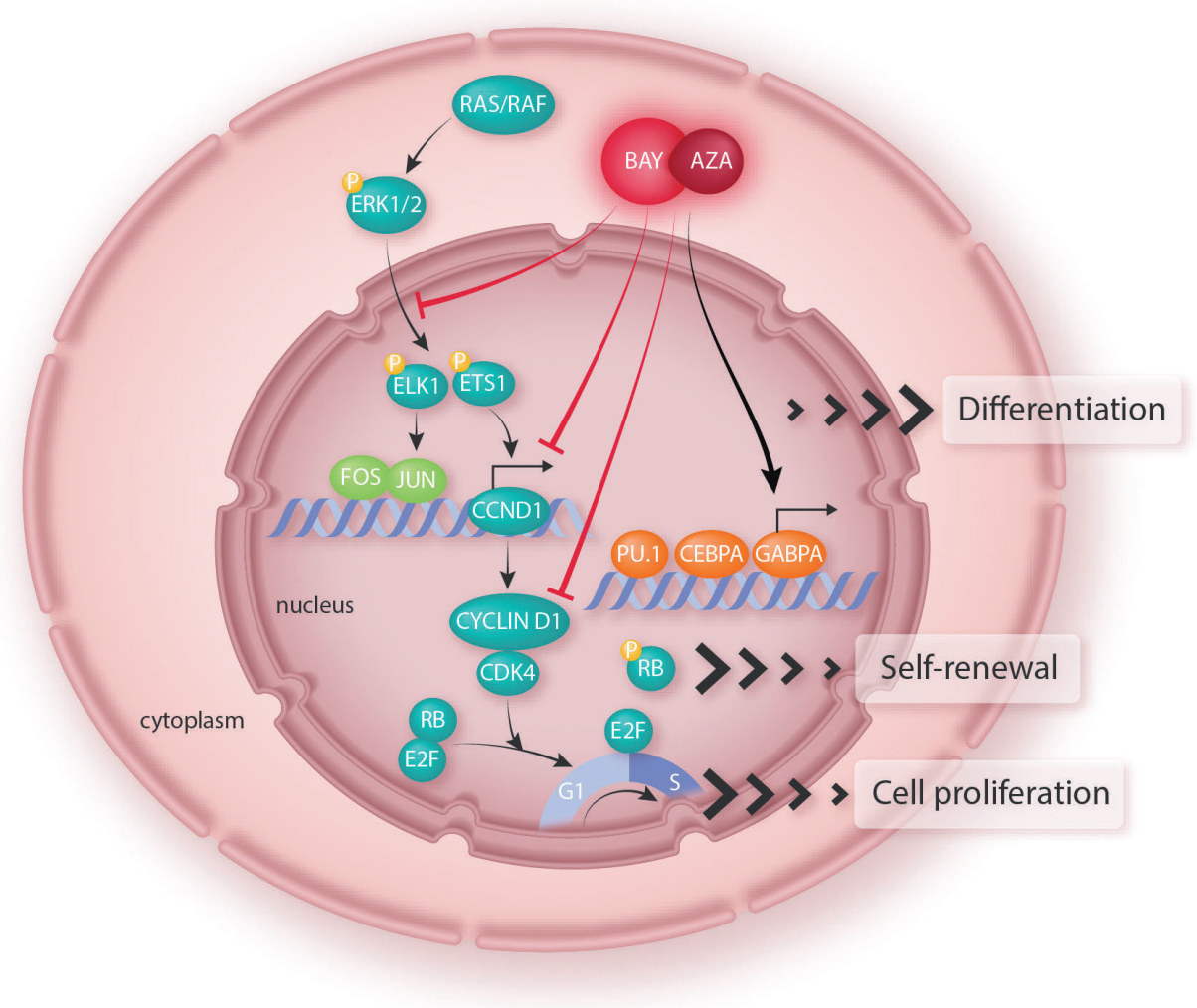

Figure 1. Schema of the molecular mechanisms of cotargeting IDH and methyltransferase in IDH mutant acute myeloid leukemia. The concurrently administered combination of the isocitrate dehydrogenase (IDH) inhibitor BAY1436032 (BAY) and the hypomethylating agent azacitidine (AZA) synergistically inhibits RAS/RAF/ERK1/2 and its downstream targets ELK1, ETS1, and CCND1 and blocks complex formation of CYCLIN D1/CDK4 to prevent RB phosphorylation, consequently inhibiting E2F release from the RB/E2F complex to promote cell cycle transition from G1 to S, leading to suppression of cell proliferation and self-renewal. In parallel, the combination of BAY and AZA upregulates the myeloid differentiation transcription factors PU.1, CEBPA, and GABPA to promote cell differentiation.

inhibited proliferation in IDH mutant AML than in IDH wild-type AML. These data support the synergistic activity of BAY1436032 and azacitidine and identify inhibition of the MAPK/RB pathways and activation of differentiation-related transcriptional factors as the key mechanisms underlying this synergism in IDH mutant AML.

Chaturvedi et al.' $\mathrm{s}^{1}$ work is important for several reasons. It is highly relevant to the ongoing clinical trials exploring combinations of IDH inhibitors and HMA, which have reported encouraging initial findings in patients with IDH mutant AML. ${ }^{13}$ These trials are designed to concurrently administer these agents, which was also confirmed in this study as being most efficacious. Furthermore, this work not only advances our understanding of the molecular regulators affected by cotargeting IDH and methyltransferase, but also hints at the complexity of the inhibitory mechanisms that can be impacted by administration sequence and contribute to differential outcomes. The authors identified the MAPK/ERKCYCLIN D1/CDK4-RB/E2F axis as critical to the regulation of LSC proliferation and the response to concurrent BAY1436032 and azacitidine. Whether this crosstalk between MAPK/ERK and RB/E2F signaling is intrinsic to IDH mutant AML, how it is associated with the terminal differentiation of LSC, and whether it can be utilized as a biomarker to predict outcomes are questions worthy of future exploration. Notably, despite its striking antileukemic activity in IDH mutant AML, the authors indicated that concurrent BAY1436032 and azacitidine failed to completely eliminate LSC. Similarly, in ongoing trials, a small fraction of patients was primary refractory or experienced AML relapse while being treated with the combination regimen of IDH inhibitor and HMA. Hypotheses to explain treatment insensitivity include incomplete mutation clearance, polyclonal resistance, and/or clonal expansion associated with activation of multiple kinases. Clearly, identification of the molecular determinants of primary and adaptive resistance is essential to refine the future therapeutic strategy. Given the genetic complexity and heterogeneity of AML, future large cohort studies and personalized molecular profiling at the singlecell level are needed to identify optimal therapeutic combinations, aiming to achieve a curative response in AML patients carrying IDH mutations.

\section{Disclosures}

No conflicts of interest to disclose.

\section{Contributions}

$Z Z$ and $M K$ wrote and edited the manuscript.

\section{References}

1. Chaturvedi A, Gupta C, Gabdoulline R, et al. Synergistic activity of IDH1 inhibitor BAY1436032 with azacitidine in IDH1 mutant acute myeloid leukemia. Haematologica. 2020;106(2):565-573.

2. Waitkus MS, Diplas BH, Yan H. Biological role and therapeutic 
potential of IDH mutations in cancer. Cancer Cell. 2018;34(2):186195.

3. Clark O, Yen K, Mellinghoff IK. Molecular pathways: isocitrate dehydrogenase mutations in cancer. Clin Cancer Res. 2016;22(8):1837-1842.

4. Cancer Genome Atlas Research N, Ley TJ, Miller C, et al. Genomic and epigenomic landscapes of adult de novo acute myeloid leukemia. N Engl J Med. 2013;368(22):2059-2074.

5. Metzeler KH, Herold T, Rothenberg-Thurley M, et al. Spectrum and prognostic relevance of driver gene mutations in acute myeloid leukemia. Blood. 2016;128(5):686-698.

6. Medeiros BC, Fathi AT, DiNardo CD, Pollyea DA, Chan SM, Swords $\mathrm{R}$. Isocitrate dehydrogenase mutations in myeloid malignancies. Leukemia. 2017;31(2):272-281.

7. Ragon BK, DiNardo CD. Targeting IDH1 and IDH2 mutations in acute myeloid leukemia. Curr Hematol Malig Rep. 2017;12(6):537546.

8. Golub D, lyengar N, Dogra S, et al. Mutant isocitrate dehydrogenase inhibitors as targeted cancer therapeutics. Front Oncol. 2019;9:417.

9. Chaturvedi A, Herbst L, Pusch S, et al. Pan-mutant-IDH1 inhibitor BAY1436032 is highly effective against human IDH1 mutant acute myeloid leukemia in vivo. Leukemia. 2017;31(10):2020-2028.

10. Heuser M, Palmisiano N, Mantzaris I, et al. Safety and efficacy of BAY1436032 in IDH1-mutant AML: phase I study results. Leukemia. 2020;34(11):2903-2913

11. Intlekofer AM, Shih AH, Wang B, et al. Acquired resistance to IDH inhibition through trans or cis dimer-interface mutations. Nature. 2018;559(7712):125-129

12. Choe S, Wang H, DiNardo CD, et al. Molecular mechanisms mediating relapse following ivosidenib monotherapy in IDH1-mutant relapsed or refractory AML. Blood Adv. 2020;4(9):1894-1905.

13. Dinardo CD, Stein AS, Stein EM, et al. Mutant IDH (mIDH) inhibitors, ivosidenib or enasidenib, with azacitidine (AZA) in patients with acute myeloid leukemia (AML). J Clin Oncol. 2018;36(15 Suppl):S7042

\section{Ironing out an approach to alleviate the hypoferremia of acute inflammation}

\section{Karin E. Finberg}

Yale School of Medicine, New Haven, CT, USA

E-mail: KARIN E. FINBERG - karin.finberg@yale.edu

doi:10.3324/haematol.2020.266627

I $\mathrm{n}$ the steady state, iron levels in the plasma are regulated by the recycling of iron from senescent red blood cells by macrophages of the reticuloendothelial system. In systemic infections and inflammatory states, perturbation of this process can result in hypoferremia (a decrease in circulating iron levels), which may represent a host defense mechanism to limit iron availability to pathogens. ${ }^{1}$ Because hypoferremia restricts the availability of iron to erythroid precursors, if sustained, it contributes to the development of the anemia of inflammation. In this issue of Haematologica, Agoro et al. ${ }^{2}$ report that the acute hypoferremic response to lipopolysaccharide (LPS), a major component of the outer membrane of Gram-negative bacteria, is modulated in mice by pretreatment with a truncated, C-terminal fragment of the hormone fibroblast growth factor 23.

During inflammatory states, iron is sequestered in cells due to a reduction in activity of ferroportin, the major cellular iron exporter that is expressed by multiple cell types, including macrophages. ${ }^{3}$ Activity of the ferroportin transporter on cell membranes is regulated by hepcidin, the key iron regulatory hormone synthesized primarily by the liver; hepcidin occludes the ferroportin transporter and triggers its endocytosis and degradation. ${ }^{4}$ Hepcidin expression is induced in response to several proinflammatory cytokines, including interleukin-6 (IL$6)$ and IL-1 $\beta$, as well as LPS. ${ }^{1}$ In addition to its post-translational regulation by hepcidin, ferroportin expression is regulated at the mRNA level by inflammatory stimuli. In macrophages, stimulation of toll-like receptor 4 (TLR4), a member of the pattern recognition receptor family, with LPS suppresses ferroportin mRNA and protein levels and also induces hepcidin expression. ${ }^{5}$ Stimulation of TLR2 and TLR6 also promotes ferroportin downregulation in a hepcidin-independent manner. ${ }^{6}$ In humans, LPS injection induces an acute rise in plasma cytokines such as IL-6 and tumor necrosis factor (TNF), which is followed by hepcidin elevation, and ultimately a reduction in serum iron levels.?

Recent studies have suggested intriguing crosstalk between inflammation, iron homeostasis, and fibroblast growth factor 23 (FGF23), ${ }^{8}$ a hormone that functions as a key regulator of phosphate and calcium homeostasis. FGF23 in the circulation appears to be mainly derived from bone, although FGF23 expression has also been detected in other tissues. ${ }^{9}$ Physiological actions of FGF23 are mediated through fibroblast growth factor receptors (FGFR) and by the co-receptor Klotho, which increases affinity of FGF23 for FGFR and is required for the hormone's ability to promote renal phosphate excretion. ${ }^{10}$ The phosphaturic activity of the mature, biologicallyactive FGF23 peptide can be abrogated by proteolytic cleavage at an RXXR motif located at the boundary between the FGF core homology domain and the 72amino-acid C-terminal portion of FGF23. ${ }^{9}$ The isolated Cterminal FGF23 fragment (referred to here as C-FGF23) competes with full-length FGF23 for binding to the FGFRKlotho complex, thereby impairing FGF23 signaling; accordingly, in healthy rodents, C-FGF23 administration inhibits renal phosphate excretion and induces hyperphosphatemia. ${ }^{11}$

Circulating FGF23 levels are markedly elevated in chronic kidney disease, ${ }^{9}$ a condition in which disruption of systemic iron homeostasis, mediated by factors such as inflammation, therapy-related iron losses, decreased glomerular filtration rate, low serum erythropoietin (EPO), and elevated serum hepcidin, contributes to the development of anemia. ${ }^{12}$ Both iron deficiency and inflammation stimulate the production of FGF23 as well as its proteolytic cleavage. ${ }^{8}$ Interestingly, in mice with established chronic kidney disease (induced by subtotal nephrectomy), a single dose of C-FGF23 induced acute 